\title{
Magnetism of free and supported vanadium clusters
}

\author{
S E Weber†, B K Raoł, P Jenał, V S Stepanyukł, W Hergert§, \\ $\mathrm{K}$ Wildberger\|, R Zeller\| and P H Dederichs\| \\ $\dagger$ Physics Department, Virginia Commonwealth University, Richmond, VA 23284-2000, USA \\ $\ddagger$ Max-Planck-Institut für Mikrostrukturphysik, Weinbergweg 2, D-06120 Halle, Germany \\ $\S$ Fachbereich Physik, Martin-Luther-Universität, Fr.-Bach-Platz 6, D-06099 Halle, Germany \\ || Institut für Festkörperforschung, Forschungszentrum Jülich, D-52425 Jülich, Germany
}

Received 28 July 1997

\begin{abstract}
Magnetic properties of free and supported Vanadium clusters of up to four atoms have been calculated self-consistently using the density functional theory. For the free clusters we have used the self-consistent field-linear combination of atomic orbitals-molecular orbital theory with a Gaussian basis for the atom. The geometries, together with the preferred spin multiplicities, were optimized by using the method of steepest descent. For supported clusters on $\mathrm{Cu}(001)$ and $\mathrm{Ag}(001)$, we have used the self-consistent Korringa-Kohn-Rostoker (KKR)-Green's function approach. Both free and supported clusters are found to be magnetic, although the magnetic moments depend strongly on the cluster size. While free clusters have ferromagnetic ground states, the supported $\mathrm{V}$ clusters in general prefer antiferromagnetic configurations. The role of inter-atomic distances, coordination, and surface morphology on the magnetic properties of $\mathrm{V}$ clusters are discussed.
\end{abstract}

\section{Introduction}

The ability of experimentalists to synthesize materials with reduced size (clusters and nanostructures) and dimension (one dimensional chains and multilayers), has given rise to a renewed interest in the study of magnetism of atomically engineered materials [1,2]. It is commonly believed that the magnetic moment per atom is enhanced when the system's size and/or dimensionality is lowered. This is because both these factors reduce the coordination number which in turn reduces the bandwidth [3]. Consequently, the density of states near the Fermi energy is enhanced causing the moments to rise. Experiments $[2,4]$ on clusters, nanostructures and ultra-thin films support this qualitative description.

The fact that reduced size and dimension can enhance magnetism of ferromagnetic materials has led to numerous investigations (both experimental and theoretical) which probe possible magnetism in otherwise non-magnetic materials [5-8]. For example, it was predicted [5,6] and later verified experimentally [7] that alkali metal clusters as well as $\mathrm{Rh}$ clusters [8] could be magnetic. Theoretical calculations of $3 \mathrm{~d}$ transition metal monolayers on metal substrates $[9,10]$ have also predicted substantial moments and magnetic ordering. Some of these predictions have been verified experimentally [1].

One of the most controversial results on magnetism of otherwise non-magnetic material in reduced size and dimension has to do with vanadium. For example, a number of theoretical calculations with varying degrees of approximation have predicted $\mathrm{V}$ monolayers to be magnetic $[10,11]$. However, there are conflicting experimental results. While spinpolarized photoemission measurements provided no evidence [12] for ferromagnetism of $\mathrm{V}$ 
on $\operatorname{Ag}(001)$, magnetism of ultrathin $\mathrm{V}$ layers sandwiched in $\mathrm{Ag}$ layers has been observed using a SQUID magnetometer [13]. Recently, experimental investigation of $\mathrm{V}$ layers on Mo substrates support the latter conclusion [14].

The study of magnetism of free V clusters also suffers from the same controversy. An early experiment by Akoh and Tasaki [15] confirmed that small particles of vanadium in the size range 100-1000 atoms are magnetic. However, a recent experiment of Douglass et al [16] found no evidence of magnetism in free $\mathrm{V}$ clusters containing as few as 9 atoms. This disagreement between two experimental results is striking, as it is well known that the properties of clusters approach bulk behaviour as their size increases. How is it possible, then, that large $\mathrm{V}$ clusters are magnetic, while small clusters are not?

The disagreement among theoretical results is less severe. A number of groups have studied the magnetism of $\mathrm{V}$ clusters containing up to 15 atoms $[3,17,18]$. These clusters were confined to the bulk bcc geometry and bulk interatomic spacing. All calculations predict that small $\mathrm{V}$ clusters are magnetic although the magnitudes of the magnetic moments differ depending on the level of approximations used. It is, however, hard to compare these results with experiments on free clusters for the following reasons: (i) The geometry of small clusters does not resemble the atomic arrangement in the corresponding bulk. (ii) The interatomic spacings in small clusters are usually less than that in the bulk. Both these factors are well known to have a significant effect on the magnetic moment.

Recently, magnetism of $\mathrm{V}$ islands on $\operatorname{Ag}(001)$ has been studied using the empirical tight-binding approach [19]. The authors find the islands to be magnetic. Furthermore, the moments depend on the nature of the coupling between the $\mathrm{V}$ atoms and the nearest neighbour sites. No experiments are available to our knowledge to compare with the above prediction.

In this paper we study the electronic structure, relative stability and magnetism of $\mathrm{V}_{n}(n \leqslant 4)$ clusters in free space as well as those deposited on $\mathrm{Ag}(001)$ and $\mathrm{Cu}(001)$ substrates. The surfaces of $\mathrm{Cu}$ and $\mathrm{Ag}$ both have the electronic d-bands located well below the Fermi level. The influence of the noble metals on clusters is mainly due to the hybridization of the cluster d-electrons with the s- and p-levels of the substrate. Our aim is to see how the interaction of clusters with substrate atoms affects their electronic and magnetic properties compared to those in free space. This understanding is important since efforts are currently being made in synthesizing cluster assembled materials by depositing them on various substrates [20]. Our calculations are performed using state-of-the-art first principles theoretical techniques. The study of free clusters is carried out using the selfconsistent field-linear combination of atomic orbitals-molecular orbital (SCF-LCAO-MO) theory [21]. The calculations on $\mathrm{V}$ clusters supported on $\mathrm{Ag}$ and $\mathrm{Cu}$ surfaces are performed using the self-consistent KKR-Green's function method [22]. Both theories make use of the spin density functional formalism. For free clusters we have used both the local spin density approximation (LSDA) as well as the generalized gradient approximation. For supported clusters, only the LSDA form is used. The studies are carried out for different spin multiplicities, magnetic coupling and geometry.

In the following section we briefly outline the theoretical procedures used for the free and supported clusters. The results are described in section 3 and summarized in section 4 .

\section{Theoretical procedure}

The theoretical techniques used for studying free and supported clusters are different and are outlined separately in the following. 


\subsection{Free clusters}

The wavefunction $\psi^{\sigma}$ for the cluster is represented by a molecular orbital constructed from a linear combination of atomic orbitals $\phi^{\sigma}$, centred at individual atomic sites $\boldsymbol{R}_{v}$, given by

$$
\psi_{i}^{\sigma}(\boldsymbol{r})=\sum_{\nu} c_{i v}^{\sigma} \phi_{\nu}^{\sigma}\left(\boldsymbol{r}-\boldsymbol{R}_{v}\right)
$$

The coefficients of linear combination $c_{i v}^{\sigma}$ are obtained by solving the following secular equation (in atomic units) self-consistently:

$$
\left(-\frac{\nabla^{2}}{2}+V_{e s}+V_{x c}^{\sigma}\right) \psi_{i}^{\sigma}(\boldsymbol{r})=E_{i}^{\sigma} \psi_{i}^{\sigma}(\boldsymbol{r}) .
$$

Here $V_{e s}$ and $V_{x c}$ are the electrostatic and exchange-correlation potentials respectively. The exchange-correlation potential was treated at two levels of theory: the local spin density approximation and the generalized gradient approximation in the density functional theory. The atomic wavefunctions $\phi^{\sigma}$ are fitted to a set of Gaussian functions and the total energy of the cluster for a fixed spin multiplicity is calculated self-consistently using the Gaussian 94 software [23]. The LSDA calculations used the exchange and correlation functional due to Vosko et al [24], while the generalized gradient approximation (GGA) made use of the Becke-Perdew-Wang method (BPW91) [25]. We have performed all electron calculations using a $(14 \mathrm{~s}, 9 \mathrm{p}, 5 \mathrm{~d} / 9 \mathrm{~s}, 5 \mathrm{p}, 3 \mathrm{~d})$ basis for $\mathrm{V}$. The forces at the atomic sites were calculated using the numerical gradient technique and the geometry is optimized using the method of steepest descent. The binding energy, $E_{b}(n)$ of a $\mathrm{V}_{n}$ cluster is defined as

$$
E_{b}=-\left[E(n)-n E_{0}\right]
$$

where $E(n)$ is the total energy of the $n$-atom cluster and $E_{0}$ is the energy of the atom. The vertical ionization potential is calculated by taking the difference between the total energies of the neutral and singly charged cluster, both having the neutral geometry.

\subsection{Supported clusters}

The calculational method used for the supported clusters is described here only briefly, since details can be found elsewhere [22]. The calculations are carried out using the KKR-Green's function method. The method is based on density functional theory in the local spin density approximation (LSDA). The Green's function of the bulk system is transformed into a layer representation. The atomic potentials of seven layers are removed to create two practically uncoupled half-crystals. The Green's function of the ideal surface is then used as the reference Green's function to calculate the electronic properties of supported clusters. The cluster on the surface destroys $2 \mathrm{~d}$ translational symmetry of the ideal surface. Therefore the Green's function of the cluster is calculated from a Dyson equation in site representation:

$$
G_{L L^{\prime}}^{n n^{\prime}}(E)=G_{L L^{\prime}}^{0 n n^{\prime}}(E)+\sum_{n^{\prime \prime} L^{\prime \prime}} G_{L L^{\prime \prime}}^{0 n n^{\prime \prime}}(E) \Delta t_{L^{\prime \prime}}^{n^{\prime \prime}}(E) G_{L^{\prime \prime} L^{\prime}}^{n^{\prime \prime} n^{\prime}}(E)
$$

Here $G_{L L^{\prime}}^{n n^{\prime}}(E)$ is the energy-dependent structural Green's function matrix and $G_{L L^{\prime}}^{0 n n^{\prime}}(E)$ the corresponding matrix for the ideal surface. The summation is over all lattice sites $n^{\prime \prime}$ and all angular momenta $L^{\prime \prime}$ for which the perturbation $\Delta t_{L^{\prime \prime}}^{n^{\prime \prime}}(E)$ between the $t$ matrices of the real and the reference system is significant. In our calculations perturbations on the nearest neighbour sites of the supported cluster are taken into account. While angular moments up to $l=3$ are included in the calculations of the wavefunction, the Coulomb and exchangecorrelation potentials were calculated using the full charge density by taking into account a multipole expansion up to $l=6$. For the latter the functional of Vosko et al [24] is used. 
Only the spherical symmetric part of the potential inside the Wigner-Seitz sphere is used to calculate the Green's function. This approximation is not important for the calculation of local moments [26].

\section{Results and discussion}

The equlibrium geometry, binding energy and preferred spin multiplicities of free $\mathrm{V}_{n}(n \leqslant 4)$ clusters were calculated using the SCF-LCAO-MO method. To test the accuracy of the method we first compare our calculated results on the ground state of the $\mathrm{V}$ atom, as well as the energetics of the $V_{2}$ dimer, with the corresponding experimental results [27]. The ground state of the $\mathrm{V}$ atom in the local spin density approximation is ${ }^{6} D_{1 / 2}$. This corresponds to a magnetic moment of $5 \mu_{B}$. However, when corrections to the exchange-correlation potential are applied, based on Becke's generalized gradient approximation (GGA), the ground state of the $\mathrm{V}$ atom using the above basis set is ${ }^{4} F_{3 / 2}$ with a moment of $3 \mu_{B}$ in agreement with experiment [27]. We have calculated the free $V$ atom also by means of our KKR technique. If we place a single $\mathrm{V}$ atom in the middle of the vacuum layers, which cuts the bulk $\mathrm{Ag}$ system into two $\operatorname{Ag}(001)$ half-crystals, we get a magnetic moment of $4.68 \mu_{B}$ in the WignerSeitz cell. This is in agreement with the LSDA version of the SCF-LCAO-MO calculation. We recall that in the KKR formalism the exchange-correlation potential is treated within the LSDA and non-integer occupancies are allowed. The small difference between $5.0 \mu_{B}$ calculated for the free atom within the SCF-LCAO-MO scheme and $4.68 \mu_{B}$ in the KKR scheme is partly due to the small influence of the $\mathrm{Ag}$ substrate in the latter method and partly due to the non-integer occupancies allowed in these calculations.

Table 1. Total energies, binding energies per atom, bond lengths and magnetic moments per atom of free $\mathrm{V}_{n}$ clusters using the LSDA and non-local correction (GGA).

\begin{tabular}{|c|c|c|c|c|c|c|}
\hline \multirow[b]{2}{*}{ Cluster } & \multicolumn{2}{|c|}{$\begin{array}{l}\text { Binding energy per atom } \\
(\mathrm{eV})\end{array}$} & \multicolumn{2}{|c|}{$\begin{array}{l}\text { Bond lengths } \\
(\AA)\end{array}$} & \multicolumn{2}{|c|}{$\begin{array}{l}\text { Magnetic moment per atom } \\
\qquad\left(\mu_{B}\right)\end{array}$} \\
\hline & GGA & LSDA & GGA & LSDA & GGA & LSDA \\
\hline $\mathrm{V}_{2}$ & 1.47 & 2.76 & 1.73 & 1.71 & 1.00 & 1.00 \\
\hline $\mathrm{V}_{3}$ & 1.76 & 2.99 & 2.14 & 2.10 & 1.67 & 1.67 \\
\hline $\begin{array}{l}\text { Equi. triangle } \\
V_{3} \\
\text { Linear }\end{array}$ & 1.26 & 2.48 & 2.05 & 1.87 & 2.33 & 2.33 \\
\hline $\begin{array}{l}\mathrm{V}_{4} \\
\text { Rhombus }\end{array}$ & 2.15 & 3.15 & $\begin{array}{l}a=2.06 \\
\alpha=114.33\end{array}$ & $\begin{array}{l}a=2.14 \\
\alpha=118.29\end{array}$ & 1.00 & 2.00 \\
\hline $\begin{array}{l}\mathrm{V}_{4} \\
\text { Tetrahedron }\end{array}$ & 2.20 & 3.57 & $\begin{array}{l}a=2.16 \\
b=2.33\end{array}$ & $\begin{array}{l}a=2.11 \\
b=2.27\end{array}$ & 1.00 & 1.00 \\
\hline
\end{tabular}

The magnetic moments of the $\mathrm{V}$ adatom (see table 2) on the $\mathrm{Cu}(001)$ and $\operatorname{Ag}(001)$ substrates are respectively $3.03 \mu_{B}$ and $3.41 \mu_{B}$ and are considerably reduced from the free atom value of $4.68 \mu_{B}$. This is caused by the hybridization of the $\mathrm{V}$ d-electrons with the sp-electrons of the metal substrates and by the nearly total quenching of the s-moment. This hybridization is stronger for the $\mathrm{Cu}(001)$ substrate than for the $\mathrm{Ag}(001)$ substrate.

The calculated ionization potential of the $\mathrm{V}$ atom is $6.12 \mathrm{eV}$ and compares well with the experimental value [27] of $6.74 \mathrm{eV}$. The electronic ground state of $\mathrm{V}_{2}$ is ${ }^{3} \sum_{g}^{-}$and agrees with recent experiment [28]. The calculated binding energy and bond length of $\mathrm{V}_{2}$ at the GGA level of theory are respectively $2.94 \mathrm{eV}$ and $1.73 \AA$. The corresponding experimental 


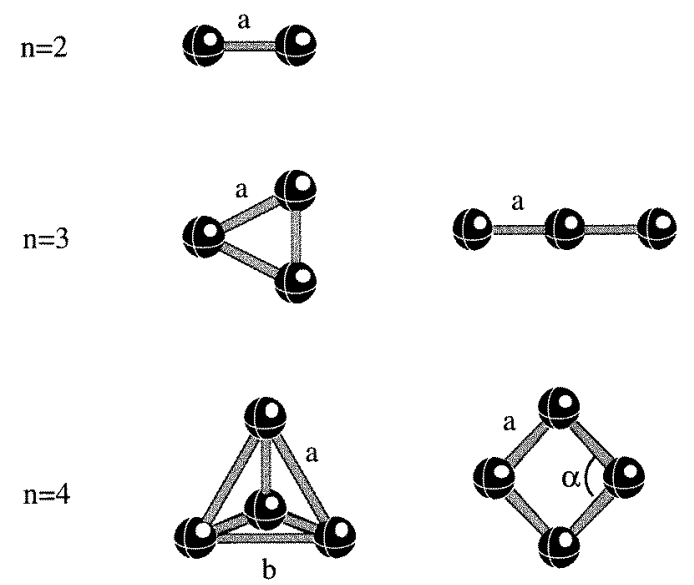

Figure 1. Equilibrium geometries of free $\mathrm{V}_{n}$ clusters. The geometrical parameters are given in table 1.

values [28] are $2.75 \mathrm{eV}$ and $1.77 \AA$. We should note that LSDA tends to overestimate the binding energy while the bond length is less sensitive. For example, we obtain a binding energy of $5.52 \mathrm{eV}$ and a bond length of $1.71 \AA$ for $\mathrm{V}_{2}$ using LSDA. Both GGA and LSDA give the ground state of $V_{2}$ to be a triplet. There have been a number of earlier theoretical works [29] on $V_{2}$ ranging from extended Hückel to local spin density calculations, which yield the ground state of $\mathrm{V}_{2}$ to be ${ }^{9} \sum_{u}^{-}$or ${ }^{1} \sum_{g}^{+}$. However, our result of spin triplet as the ground state of $\mathrm{V}_{2}$ agrees with recent experiment [28] and theory [3,30].

The geometries of the $\mathrm{V}_{n}$ clusters are given in figure 1 . The bond lengths, binding energies, and the magnetic moments per atom are given in table 1 . Note that, as in $\mathrm{V}_{2}$, the binding energy per atom in the local spin density approximation is consistently higher than that using the gradient correction. However, the bond lengths, bond angles and the magnetic moment per atom remain practically unaffected. It is also interesting to note that the binding energy of $1.47 \mathrm{eV} /$ atom and bond length of $1.73 \AA$ of the $\mathrm{V}_{2}$ dimer are significantly less than the corresponding values for bulk vanadium, which has a cohesive energy of $5.31 \mathrm{eV}$ and a nearest neighbour distance of $2.62 \AA$. The magnetic moment per atom of the dimer is $1.0 \mu_{B}$ and is significantly reduced from the gradient corrected free atom value of $3.0 \mu_{B}$.

To aid in the discussion to follow, we have calculated the binding energy of a $V_{2}$ dimer as a function of interatomic distance for different spin multiplicities, such as triplet, quintet, septet, etc. It is expected that as the interatomic separation of the $V_{2}$ dimer is increased, the overlap between the orbitals centred at each atomic site should decrease. This in turn should cause the magnetic moment per atom to increase. To examine how this process evolves, we plot the binding energy (calculated using the GGA) of the dimer in figure 2 for various spin multiplicities. Note that when the interatomic separation reaches about $1.9 \AA$, the ground state changes from a triplet to quintet. At an interatomic distance of about $2.2 \AA$, the states with spin multiplicities of 5 and 7 are nearly degenerate. Beyond this distance the preferred spin multiplicity is 7 . Thus, as $\mathrm{V}$ clusters are deposited on a metallic substrate, their interaction with the substrate atoms will cause the $\mathrm{V}-\mathrm{V}$ distance to conform to the distance in the underlying lattice. Consequently, this factor alone can cause their magnetic moment to differ from the free cluster value.

For example, when we confine the $\mathrm{V}_{2}$ dimer to a distance of $2.56 \AA$ and $2.89 \AA$, 


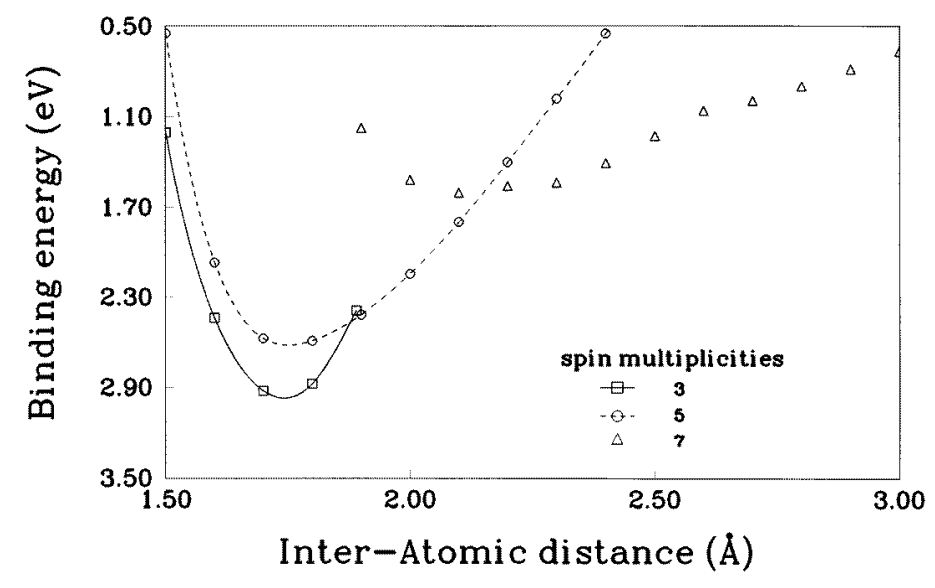

Figure 2. Binding energies $E_{b}$ for the $\mathrm{V}_{2}$ dimer as a function of interatomic distance and spin multiplicities. Note that the energies are based on the generalized gradient approximation in the density functional theory.

Table 2. Magnetic moments of ferromagnetic states of $\mathrm{V}_{n}$ clusters on $\mathrm{Cu}(001)$ and $\mathrm{Ag}(001)$ surfaces ( $\mathrm{C} n$-chains of $n$ atoms, I4-island of 4 atoms). For the trimer $\mathrm{C} 3$, having two nonequivalent sites, only the average moment per atom is given.

\begin{tabular}{llll}
\hline Cluster & Position & $\begin{array}{l}\mathrm{Cu}(001) \\
\text { Magnetic moment per atom } \\
\left(\mu_{B}\right)\end{array}$ & $\begin{array}{l}\operatorname{Ag}(001) \\
\text { Magnetic moment per atom } \\
\left(\mu_{B}\right)\end{array}$ \\
\hline $\mathrm{C} 1$ & Free atom & 4.68 & 4.68 \\
& Adsorbate atom & 3.03 & 3.41 \\
& Terrace atom & 2.46 & 3.06 \\
$\mathrm{C} 2$ & Bulk impurity [10] & 1.10 & 2.89 \\
& Free dimer & 3.45 & 3.58 \\
& Adsorbate dimer & 2.85 & 3.38 \\
$\mathrm{C} 3$ & Bulk dimer [10] & 0.00 & 2.81 \\
$\mathrm{I} 4$ & Adchain & 2.76 & 3.36 \\
\hline
\end{tabular}

which corresponds to distances the dimers would have on a $\mathrm{Cu}(001)$ and $\operatorname{Ag}(001)$ surface, respectively, the magnetic moment per atom is $3.0 \mu_{B}$. Using the KKR formalism we have calculated the magnetic moment per atom of $\mathrm{V}_{2}$ on the $\mathrm{Cu}(001)$ and $\mathrm{Ag}(001)$ surfaces as well as in the bulk systems. In order to check the accuracy of the method, we have also used the KKR method to simulate the free $\mathrm{V}_{2}$ dimer as was done for the free $\mathrm{V}$ atom in the discussion above. Our calculated values of $3.45 \mu_{B} /$ atom for the $\mathrm{Cu}(001)$ and $3.58 \mu_{B} /$ atom for the $\mathrm{Ag}(001)$ surfaces agree well with the spin septet configuration obtained in the SCFLCAO-MO method (see table 2). Note again that the KKR method only used the LSDA level of theory while our results in figure 2 are with generalized gradient approximation. However, the use of the LSDA (as opposed to the GGA) method in the KKR calculation is not expected to adversely affect our conclusions concerning the magnetic moments of the supported V clusters. As mentioned earlier and shown in table 1, the magnetic moments are not sensitive to these approximations in clusters.

The interaction of the dimer with the Ag surface reduces the moment to $3.38 \mu_{B}$ per 
atom. This is mostly due to the further quenching of the s-moments on the surfaces. The smaller interatomic spacing on the $\mathrm{Cu}(001)$ surface compared to that of $\mathrm{Ag}$ and a stronger hybridization of the $\mathrm{V}$ wavefunctions with the sp-wavefunctions of the $\mathrm{Cu}$ substrate reduces the moment of the supported $\mathrm{V}_{2}$ dimer further to $2.85 \mu_{B}$. Blügel et al [10] have also calculated the moments of single $\mathrm{V}$ impurities and $\mathrm{V}$ impurity pairs in bulk $\mathrm{Cu}$ and $\mathrm{Ag}$. In this paper it was shown that the strength of the $3 d-3 d$ hybridization increases with the delocalization of the $3 \mathrm{~d}$ wavefunction, due to the reduction of the dimer bond length. Our results on the relative variation of magnetic moments of $\mathrm{V}_{2}$ dimers on $\mathrm{Cu}$ and $\mathrm{Ag}$ are consistent with this study.

The structure of the free $\mathrm{V}_{3}$ trimer is an equilateral triangle with a bond length of $2.14 \AA$ and a binding energy of $1.76 \mathrm{eV} /$ atom. The magnetic moment per atom is $1.67 \mu_{B}$ and is significantly higher than the dimer value of $1.0 \mu_{B}$. To understand the origin of this increase, we note that the interatomic distance as well as the coordination number in $\mathrm{V}_{3}$ are larger than those in $\mathrm{V}_{2}$. Typically these two factors act in opposite directions-increasing the interatomic distance causes the moments to rise (as seen in figure 2), while increasing the coordination number causes the moments to decrease. If $\mathrm{V}_{2}$ is constrained to a bond length of $2.14 \AA$, the preferred moment per atom for this configuration would be $2 \mu_{B} /$ atom. The fact that in $\mathrm{V}_{3}$ the magnetic moment is $1.67 \mu_{B}$ /atom shows that the increasing coordination has played a role in diminishing the magnetic moment.

Brune et al [2] have shown that small linear chains and planar islands can be created on a substrate by special growth techniques. Therefore, we have studied the magnetic moments of a $V_{3}$ linear chain and $V_{4}$ in-plane square island configuration. Other geometries are possible, but here we only restrict ourselves to the above geometries.

The moments of the supported trimers are changed only slightly $\left(2.76 \mu_{B}\right.$ for $\mathrm{Cu}(001)$ and $3.36 \mu_{B}$ for $\left.\operatorname{Ag}(001)\right)$ compared with the dimers. The atom in the middle of the trimer has a higher coordination and therefore a smaller moment $\left(2.58 \mu_{B}\right.$ for $\mathrm{Cu}(001)$ and $3.34 \mu_{B}$ for $\mathrm{Ag}(001))$ than the other two atoms $\left(2.85 \mu_{B}\right.$ for $\mathrm{Cu}(001)$ and $3.38 \mu_{B}$ for $\left.\mathrm{Ag}(001)\right)$. This leads to a decrease of the mean value of the trimer moment (see table 2).

We have also calculated the moment of the $\mathrm{V}_{3}$ trimer as a linear chain in free space. We find that there are two magnetic solutions with $2.33 \mu_{B}$ /atom and $1.0 \mu_{B} /$ atom that are nearly degenerate, the former being approximately $0.01 \mathrm{eV}$ lower in energy. This configuration, however, is $1.5 \mathrm{eV}$ higher in energy than the groundstate, which is an equilateral triangle.

For a $\mathrm{V}_{4}$ cluster, the equilibrium structure is a slightly distorted tetrahedron. The interatomic distance in the plane is $2.33 \AA$ while that between the apex atom and an atom in the plane is $2.16 \AA$. The binding energy per atom and the magnetic moment per atom are respectively $2.20 \mathrm{eV}$ and $1.0 \mu_{B}$. The sharp decrease in the moment in $\mathrm{V}_{4}$ compared to that in $\mathrm{V}_{3}$ can be attributed to be primarily due to an increasing coordination number since the interatomic distances between $\mathrm{V}_{3}$ and $\mathrm{V}_{4}$ clusters differ very little. We have also searched for the existence of other structural isomers and found that $\mathrm{V}_{4}$ can exist as a planar rhombus structure with an interatomic distance of $2.06 \AA$. However, this structure lies $0.21 \mathrm{eV}$ in energy above the tetrahedral configuration. Interestingly, both the rhombus and tetrahedral structure have the same moment per atom at the GGA level of theory.

For the supported islands consisting of 4 atoms the moments decrease compared to the trimers. The decrease for the $\mathrm{Cu}$ substrate is similar to what has been observed for the free clusters. For the four-atom cluster on the Ag substrate the relative decrease is considerably smaller. The decrease of the moment on $\mathrm{Cu}$ is stronger than that on $\mathrm{Ag}$, since the lattice constant of $\mathrm{Cu}$ is about $10 \%$ smaller than that of $\mathrm{Ag}$ and therefore the $\mathrm{d}-\mathrm{d}$ hybridization is stronger.

It should be noted that in the supported clusters, the effect of relaxation was ignored. For 
example, the atomic positions in $\mathrm{Cu}(001)$ and $\mathrm{Ag}(001)$ substrates were assigned their ideal lattice configurations. When $\mathrm{V}$ clusters were deposited on these substrates, their positions were also assumed to conform to the ideal substrate structure, i.e. the $\mathrm{V}-\mathrm{V}$ distance and height of the cluster plane from the surface were the same as that of the substrate. This assumption was necessary due to the computational limitations. The loss of symmetry due to the absorbed clusters already makes the ab initio KKR calculations very complex. Adding the effect of surface relaxation is beyond the scope of present day computational resources if calculations at the first principles level of theory, taking into account the semiinfinite surface, are desired. However, we can study the effect of relaxation qualitatively by referring to our results in figure 2 . Note that the nearest neighbour distances in bulk $\mathrm{V}, \mathrm{Cu}$, and $\mathrm{Ag}$ are respectively $2.62,2.56$ and $2.89 \AA$. Thus, for $\mathrm{V}_{n}$ on $\mathrm{Cu}(001)$ we do not expect any significant change in the moment due to relaxation. For $\mathrm{V}_{n}$ on $\operatorname{Ag}(001)$ the lattice mismatch is about $10 \%$. As can be seen from figure 2, the moment per atom of the $\mathrm{V}_{2}$ dimer does not change for $2.6<R<2.9 \AA$. Consequently we expect the moment of $\mathrm{V}_{n}$ clusters on $\mathrm{Ag}$ to be unaffected due to relaxation as well. This expectation, of course, clearly neglects any electronic considerations arising from the interaction between the clusters and the substrates. We are currently working on methods that combine KKR with the tight binding model, which will enable us to examine, in the future, the role of surface relaxation on cluster magnetism.

In this paper we have restricted our studies of $\mathrm{V}_{n}$ clusters to the ferromagnetic solutions. This is because the $\mathrm{V}_{n}$ clusters in free space are found to be ferromagnetic. However, this seems not to be the case for the supported V clusters. Detailed calculations for $\mathrm{Cu}(001)$ [33] show that the $\mathrm{V}$ dimers, trimers and tetramers prefer antiferromagnetic configurations. For instance, the antiferromagnetic configuration of the $\mathrm{V}_{2}$ dimer is $0.2 \mathrm{eV} /$ atom lower in energy than the ferromagnetic one, and also for the $\mathrm{V}$ trimer and tetramer the configurations with antiferromagnetic nearest-neighbour coupling are lower in energy than the ferromagnetic solutions, in the case of $\mathrm{V}_{3}$ by $0.11 \mathrm{eV} /$ atom and $\mathrm{V}_{4}$ by $0.05 \mathrm{eV} /$ atom. Since all free $\mathrm{V}$ clusters are ferromagnetic, we believe that the preference of the antiferromagnetic configuration is a typical result of the hybridization with the substrate. To show this, we have calculated the ferromagnetic and antiferromagnetic state of the free $\mathrm{V}$ dimer with a bond length corresponding to the nearest neighbour distance of the Ag lattice using the KKR method. We get moments of $3.58 \mu_{B}$ for the ferromagnetic state and $\pm 3.58 \mu_{B}$ for the antiferromagnetic state. The ferromagnetic groundstate is $0.013 \mathrm{eV} /$ atom lower in energy than the antiferromagnetic state. This is in line with calculations by Blügel et al [10] for complete $\mathrm{V}$ monolayers. While a $\mathrm{V}$ monolayer deposited on $\operatorname{Ag}(001)$ prefers the antiferromagnetic configuration, a free-standing $\mathrm{V}$ monolayer with the $\mathrm{Ag}$ lattice constant shows a ferromagnetic ground state. As explained in detail by Blügel et al [10] the hybridization with the substrate slightly broadens the density of states of the monolayers, which is unfavourable for the ferromagnetic configuration.

Another characteristic property of the multimers on surfaces is the existence of multiple spin configurations. This effect has been known to exist for bulk metals, alloys, as well as ultrathin films [31]. Even some clusters exhibit multiple spin configurations. For example, the anomalous photo-detachment spectra of the $\mathrm{Li}_{4}^{-}$cluster was explained due to the coexistence of the anionic cluster in doublet and quartet spin configurations [32]. Recently Stepanyuk et al [33] have studied the energetics of the trimers of $3 \mathrm{~d}$ elements on $\mathrm{Cu}(001)$. For all $3 \mathrm{~d}$ trimers they found the existence of four different spin configurations: ferromagnetic, antiferromagnetic and two different kind of low spin states. In some particular cases such as $\mathrm{V}_{3}$ and $\mathrm{Mn}_{3}$, the energy differences between various magnetic states can be as small as $8 \mathrm{meV}$ and magnetic fluctuations are possible. In general also 
non-collinear states cannot be excluded.

The effect of the matrix on the magnetic properties of the transition metal cluster will undoubtedly be an important topic for further studies, as the possibility of synthesizing cluster assembled materials becomes promising. Thus an in depth understanding of the properties of the free and supported clusters will lead to a rich and interesting field of research in the future.

\section{Conclusions}

Self-consistent calculations based on the local spin density functional theory have been carried out to study the magnetic properties of free and supported $\mathrm{V}$ clusters on $\mathrm{Cu}(001)$ and $\operatorname{Ag}(001)$ substrates. The geometries of the free clusters were fully optimized. All free $\mathrm{V}_{n}$ clusters up to $n=4$ were found to be magnetic with moments per atom varying nonmonotonically with size. While the non-local correction to the density functional theory had a substantial effect on the magnetic moment of the free $\mathrm{V}$ atom, it had no effect on the moment of $\mathrm{V}$ clusters when compared to the local approximation. We recall that $\mathrm{V}_{n}$ clusters containing more than nine atoms were found to be magnetic experimentally. In this work we have not optimized geometries of $\mathrm{V}_{n}$ clusters for $n>4$. Thus it is difficult to compare the theory with experiment. However, we are confident about our results that $\mathrm{V}_{n}$ $(n \leqslant 4)$ clusters are magnetic. This confidence is based on the past success of the density functional theory not only predicting [6] magnetism of clusters that later were verified experimentally [8], but also in correctly identifying the ground state spin multiplicity of $\mathrm{Fe}$, $\mathrm{Co}$, and Ni dimers [34].

Surprisingly the moments of the supported V clusters are considerably larger than the ones for the free clusters. This enhancement of the moments of the supported clusters is basically due to the fact that the nearest neighbour distances offered by the $\operatorname{Ag}(001)$ and $\mathrm{Cu}(001)$ substrates are significantly larger than the corresponding distances in the free clusters. The somewhat smaller moments on the $\mathrm{Cu}$ substrate as compared to the $\mathrm{Ag}$ can also be understood in this way since the lattice constant of $\mathrm{Ag}$ is $10 \%$ larger than that of $\mathrm{Cu}$, resulting in a much reduced hybridization. Accurate description of the substrate relaxation resulting from cluster deposition is necessary before one can conclusively state that the supported $\mathrm{V}_{n}$ clusters would retain their magnetic character.

A second difference between the examined free and supported $\mathrm{V}$ clusters is the magnetic character of the ground state. While the free clusters have ferromagnetic ground states, the supported V clusters in general prefer antiferromagnetic configurations. The hybridization with the substrate leads to a broadening of the density of states, which tends to destabilize the ferromagnetic coupling. Our calculations also suggest the existence of multiple magnetic solutions. In special cases this might make it difficult to compare theory with existing experiment. Thus magnetic experiments on controlled substrates with well defined clusters would be very helpful.

\section{Acknowledgments}

This work is supported in part by the Army Research office. We than S Blügel and J Kirschner for helpful discussions. The computations were performed partially on Cray computers of the Forschungszentrum Jülich and the German supercomputer centre (HLRZ). One of the authors (PJ) is grateful to Professor K V Rao for suggesting this problem. 


\section{References}

[1] Heinrich B and Cochran J F 1993 Adv. Phys. 42523

[2] Brune H, Röder H, Boragno C and Kern K 1994 Phys. Rev. Lett. 731955

Röder H, Hahn E, Brune H, Bucher J P and Kern K 1993 Nature 366141

[3] Liu F, Khanna S N and Jena P 1991 Phys. Rev. B 438179

[4] Apsel S E, Emmert J W, Deng J and Bloomfield L A 1996 Phys. Rev. Lett. 761441

Billas I M L, Chatelain A and de Heer W A 1994 Science 2651682

Shi J, Gider S, Babcock K and Awschalom D D 1996 Science 271937

[5] Rao B K, Jena P and Manninen M 1985 Phys. Rev. B 32477

[6] Reddy B V, Khanna S N and Dunlap B I 1993 Phys. Rev. Lett. 703323

[7] Nozue Y, Kodaira T and Goto T 1992 Phys. Rev. Lett. 683789

[8] Cox A J, Louderback J G and Bloomfield L A 1993 Phys. Rev. Lett. 71923

[9] Ohnishi S, Freeman A J and Weinert M 1983 Phys. Rev. B 286741

[10] Blügel S, Drittler B, Zeller R and Dederichs P H 1989 J. Appl. Phys. A 49547

[11] Fu C L, Freeman A J and Oguchi T 1985 Phys. Rev. Lett. 542700

Gay J G and Richter R 1985 Phys. Rev. Lett. 562728

Mokrani A, Dreyssé H, Bouarab S and Demangeat C 1992 J. Magn. Magn. Mater. 113201

[12] Stampanoni M, Vaterlaus A, Pescia D, Aeschlimann M, Meier F, Dürr W and Blügel S 1988 Phys. Rev. B 3710380

[13] Shintaku K, Mizutani T, Hosoito N and Shinjo T 1991 J. Phys. Soc. Japan 601078

[14] Korenivski V, Rao K V, Birch J and Sundgren J E 1995 J. Magn. Magn. Mater. 140-144 549

[15] Akoh H and Tasaki A 1978 J. Appl. Phys. 491410

[16] Douglass D C, Boucher J P and Bloomfield L A 1992 Phys. Rev. B 456341

[17] Salahub D R and Messmer R P 1981 Surf. Sci. 106415

[18] Lee K and Callaway J 1993 Phys. Rev. B 4915358 Lee K and Callaway J 1994 Phys. Rev. B 4913906

[19] Nait-Laziz H, Demangeat C and Mokrani A 1993 J. Magn. Magn. Mater. 121123

[20] Dupuis V, Perez J P, Tuaillon J, Pillard V, Mellinon P, Perez A, Barbara B, Thomas L, Fayeulle S and Gay J M 1994 J. Appl. Phys. 766676

[21] Here W J, Random L, Schleyer P V R and Pople J A 1986 Ab initio Molecular Orbital Theory (New York: Wiley)

[22] Zeller R, Lang P, Drittler B and Dederichs P H 1992 MRS Symp. Proc. 253 (Pittsburgh, PA: Materials Research Society) p 357

Stepanyuk V S, Lang P, Wildberger K, Zeller R and Dederichs P H 1994 Surf. Rev. Lett. 1477

Lang P, Stepanyuk V S, Wildberger K, Zeller R and Dederichs P H 1994 Solid State Commun. 92755

[23] Frisch M J 1995 Gaussian 94 Revision B.1 Gaussian Inc., Pittsburgh, PA, USA

[24] Vosko S H, Wilk L and Nusair M 1980 Can. J. Phys. 581200

[25] Becke A D 1988 Phys. Rev. A 383098

Perdew J P and Wang Y 1992 Phys. Rev. B 4513244

[26] Wildberger K, Stepanyuk V S, Lang P, Zeller R and Dederichs P H 1995 Phys. Rev. Lett. 75509 Stepanyuk V S, Hergert W, Wildberger K, Zeller R and Dederichs P H 1996 Phys. Rev. B 532121

[27] Moore C E 1970 Ionization Potentials and Ionization Limits Derived from the Analyses of Optical Spectra NSRDS-NBS34 (Washington DC: National Bureau of Standards) Morse M D 1986 Chem. Rev. 861049

[28] Spain E M and Morse M D 1992 J. Phys. Chem. 962477

[29] Cooper W F, Clarke G A and Hare C R 1972 J. Phys. Chem. 762268

Ford T A, Huber K, Klotzbücher W, Kündig E P, Moskovits M and Ozin G A 1977 J. Chem. Phys. 66524 Harris J and Jones R O 1979 J. Chem. Phys. 70830

[30] Salahub D R 1987 Ab initio Methods in Quantum Chemistry II ed K P Lawley (New York: Wiley) p 447 and references therein

[31] Wassermann E F 1991 J. Magn. Magn. Mater. 100346

[32] Rao B K, Jena P and Ray A K 1996 Phys. Rev. Lett. 762878

[33] Stepanyuk V S, Hergert W, Wildberger K, Zeller R and Dederichs P H 1997 submitted

[34] Castro M, Jamorski C and Salahub D R 1997 Chem. Phys. Lett. 271133 